\title{
Electrode Material Containing Graphite Incorporated to an Amino-Functionalized Polydimethylsiloxane Network for the Detection of Copper
}

\author{
Ricardo P. D. Silva, ${ }^{a}$ Alzira M. S. Lucho ${ }^{a}$ and Fabio L. Pissetti ${ }^{* a}$ \\ ${ }^{a}$ Instituto de Química, Universidade Federal de Alfenas (UNIFAL-MG), \\ Rua Gabriel Monteiro da Silva, 700, 37130-000 Alfenas-MG, Brazil
}

\begin{abstract}
Graphite was incorporated to a polydimethylsiloxane (PDMS) polymer network functionalized with amino groups to obtain an electrode material and used to detect copper ions in aqueous solutions and sugarcane spirit. The polymer network was prepared with PDMS, 3-aminopropyltrimethoxysilane (APTMS), tetraethyl orthosilicate (TEOS) with addition of graphite to obtain the electrode material. The materials were prepared with different mass concentrations of PDMS: 0, 3, 6, 12 and 18\%; and characterized by thermogravimetric analysis (TGA) and Fourier transform infrared spectroscopy (FTIR). Quasi-reversible behavior was found when studying the $\left[\mathrm{Fe}(\mathrm{CN})_{6}\right]^{3-4-}$ redox probe and were electrochemically stable up to 100 consecutive cycles. Electrochemical impedance measurements showed a second time constant at high frequencies for the PDMS electrodes, indicating a second phase in the polymer network with resistive properties, probably due to an increase in the material polymerization resistance. Square wave voltammetry studies were conducted with these electrodes to detect copper(II) ions in aqueous solution. The electrode proposed was applied to determine $\mathrm{Cu}^{2+}$ in a real sample, sugarcane spirit. The PDMS electrodes prepared here presented a potential to be applied as electrochemical sensors for detecting copper ions.
\end{abstract} sensor

Keywords: polydimethylsiloxane, amino group, copper ion, sugarcane spirit, electrochemical

\section{Introduction}

Chemically modified electrodes (CME) are made by adding chemically active species to the surface of an electrode substrate for the purpose of pre-establishing and controlling the physicochemical nature of the electrode/ solution interface. The modification of an electrode normally gives the physicochemical characteristics (reactivity, selectivity, etc.) of the modifier. This enables the development of devices with suitable responses for many purposes and applications. ${ }^{1}$

An important aspect in the preparation of a CME is the choice of the electrode base material that will have its surface modified. This substrate should have appropriate electrochemical characteristics and be compatible with the chosen modification method, among the conventionally used materials are: gold, platinum, vitreous carbon and carbon paste. ${ }^{2}$ As an example, a modified carbon paste electrode can be made by the addition of different classes of chemical compounds to a mix of graphite powder and an agglutinative agent. ${ }^{3-5}$

*e-mail: fabio.pissetti@unifal-mg.edu.br, flpissetti@gmail.com
Polysiloxanes or silicones, considered semi-inorganic polymers, are studied in the materials field, being used even on a commercial scale. The polymer displays properties that are different from organic polymers. ${ }^{6-8}$ Most polyorganosiloxanes are of variable-viscosity fluids due to their relatively small intermolecular interactions caused by the methyl groups in the main chain of these polymers. Chain reticulation is necessary to obtain a polymeric network from polyorganosiloxanes. Synthesis of such polymeric networks may be carried out in many ways. One of the main methods used for their preparation is based on a condensation reaction of the reticulating agents with terminal silanol groups in the chain of polydimethylsiloxane (PDMS). ${ }^{9-12}$ There are literature reports ${ }^{13,14}$ on the use of PDMS in electrodes as the substrate base for chips or sensors and as coatings for solid electrode surfaces. Nevertheless, there are no reports of PDMS used as bulk component of an electrode material.

Materials functionalized with an amino group $\left(-\mathrm{NH}_{2}\right)$ have been prominently used in CMEs because this functional group promotes metal ion adsorption due to the nature as a hard base that interacts efficiently with cations classified as hard or intermediate acids. ${ }^{15,16}$ Two examples 
of this type of electrode prepared with an amino functional group are a glucose sensor obtained by modification of chitosan with 3-aminopropyltrimethoxysilane and further adsorption of ferrocene, ${ }^{17}$ and a modified silica with 3 -aminopropyltrimethoxysilane and thiosalicylic acid that was efficiently applied to determining lead. ${ }^{18}$

Industrial activity has contributed substantially to a significant increase in the metal ion concentration in effluents and represents an important source of contamination in aquatic bodies, especially because these ions are usually disseminated via the food chain. Industrial liquid effluents have a rather complex chemical composition, containing organic and inorganic compounds. ${ }^{19}$ Copper is an essential metal in living organisms; it is estimated that in a healthy human diet two to five milligrams of copper are necessary every day. Copper is distributed all over the human body because it integrates with several important enzymes that are involved in vital biological processes. Small alterations of the metal concentration in the body are insignificant, and do not lead to serious complications; however, exposure to high concentrations of metal even for a short period may cause a variety of diseases and, in more severe cases, death. ${ }^{20}$

In this study, the aim was to use PDMS as substrate to obtain a polymeric network functionalized with amino groups, which was used as chemical modifier for a graphite electrode, without necessity to add an agglutinative agent addition. Materials with different amounts of PDMS were prepared and characterized by thermogravimetric analysis (TGA) and Fourier transform infrared spectroscopy (FTIR). The conductive properties of the electrodes were assessed by cyclic voltammetry $(\mathrm{CV})$ using $\left[\mathrm{Fe}(\mathrm{CN})_{6}\right]^{3-14-}$ as a probe molecule, electrochemical stability studies were also performed and the effect of two different supporting electrolytes on the electrochemical response was investigated. In addition, electrochemical impedance measurements were performed, the electrodes were used to detect copper(II) cations in aqueous solution with square wave voltammetry measurements and in an analytical determination of real sample, an artisanal sugarcane spirit.

\section{Experimental}

A condensation process was used to prepare a conductive material from polydimethylsiloxane (PDMS), a functional silane (3-aminopropyltrimethoxysilane, APTMS), ${ }^{21}$ and the alkoxide tetraethyl orthosilicate (TEOS), with the incorporation of amorphous graphite (99.99\%). All syntheses were performed at ambient temperature. All reagents were purchased from Sigma-Aldrich ${ }^{\circledR}$ and used without subsequent purification.

\section{Preparation of the electrode material}

The material composition was previously studied varying from 25 to $75 \%$ of graphite incorporation. It was verified that the better results were obtained for the material with a proportion of $50 \%$ in mass of graphite. Thus, this ratio was used during all the further synthesis. Table 1 shows the amounts of PDMS, TEOS, APTMS and graphite used in the reaction mixtures. The preparation protocol was as follows: the reagents were added to a beaker with $5 \mathrm{~mL}$ of ethanol and homogenized by magnetic stirring for approximately $120 \mathrm{~min}$ until the viscosity increased and a solid paste was formed. The material was then pulverized and immersed in tetrahydrofuran for $1 \mathrm{~h}$; afterwards, it was dried out in a vacuum oven for approximately $12 \mathrm{~h}$ at $60^{\circ} \mathrm{C}$.

\section{Preparation of the working electrodes}

The electrodes were prepared by manual trituration of the material obtained. $30 \mathrm{mg}$ of compound was added to a glass tube of $0.1 \mathrm{~cm}$ depth, containing a platinum disc of $5 \mathrm{~mm}$ diameter (final electrode dimension) in electrical contact with a copper wire. The detail of the E6 composite is illustrated in Figure S1 (Supplementary Information (SI) section). The electrodes were named according to the percentage of PDMS in their composition using the acronyms shown in Table 1.

Table 1. Mass (absolute and percentage) of the precursors used in the preparation of different electrode materials

\begin{tabular}{lcccc}
\hline Electrode & PDMS / g & TEOS / g & APTMS / g & Graphite / g \\
\hline E0 & - & $1.25(25 \%)$ & $1.25(25 \%)$ & $2.5(50 \%)$ \\
E3 & $0.15(3 \%)$ & $1.175(23.5 \%)$ & $1.175(23.5 \%)$ & $2.5(50 \%)$ \\
E6 & $0.3(6 \%)$ & $1.1(22 \%)$ & $1.1(22 \%)$ & $2.5(50 \%)$ \\
E12 & $0.6(12 \%)$ & $0.95(19 \%)$ & $0.95(19 \%)$ & $2.5(50 \%)$ \\
E18 & $0.9(18 \%)$ & $0.8(16 \%)$ & $0.8(16 \%)$ & $2.5(50 \%)$ \\
\hline
\end{tabular}

PDMS: polydimethylsiloxane; TEOS: tetraethyl orthosilicate; APTMS: 3-aminopropyl-trimethoxysilane. 
Infrared absorption spectroscopy (FTIR)

The prepared materials were analyzed in a Shimadzu Prestige spectrophotometer using $\mathrm{KBr}$ tablets containing between 0.5 and $1 \%$ in mass concentration of the relevant material. A $4 \mathrm{~cm}^{-1}$ resolution, 50 sweeps, and a sweeping range of 4000 to $400 \mathrm{~cm}^{-1}$ were used.

\section{Thermogravimetric analysis}

Thermogravimetric curves were obtained using a TA Instruments SDT Q600 analyzer. The analyses were carried out at a $20{ }^{\circ} \mathrm{C} \mathrm{min}^{-1}$ heating speed in a $\mathrm{N}_{2}\left(100 \mathrm{~mL} \mathrm{~min}{ }^{-1}\right)$ atmosphere.

\section{Scanning electron microscopy (SEM)}

Scanning electron microscopy (SEM) images were obtained with a JEOL microscope FEG-SEM JSM 6330F operated at $5 \mathrm{kV}$. The samples for SEM were prepared by drop-casting an aqueous suspension of the composites over a Si wafer, followed by drying in air.

\section{Electrochemical characterization}

The electrochemical characterization was carried out using the PDMS electrode materials as the working electrode, a saturated calomel electrode (SCE) as the reference electrode, and a platinum wire as the counter electrode. Unless otherwise stated, an aqueous solution of $0.1 \mathrm{~mol} \mathrm{~L}^{-1} \mathrm{KCl}$ was used as the supporting electrolyte in all electrochemical experiments.

The electrochemical measurements were made using an Autolab® PGSTAT 128N (Eco Chemie) potentiostatgalvanostat. Processing and management of obtained data was performed using GPES (General Purpose Electrochemical System) software. Electrochemical impedance spectroscopy measurements were performed over a frequency range of $500 \mathrm{kHz}$ to $20 \mathrm{MHz}$, and a $5 \mathrm{mV}$ sine voltage width. The electrochemical impedance measurements were performed in open circuit potential (OCP). The acquisition and analysis of data were carried out using FRA (Frequency Response Analysis system) software.

Cyclic voltammetry studies were carried out using either $\mathrm{KCl}$ or $\mathrm{NaCl}$ supporting electrolyte, both $0.1 \mathrm{~mol} \mathrm{~L}^{-1}$. Measurements were made over a potential range of -0.25 to $1.0 \mathrm{~V}(\mathrm{SCE})$ at a scan rate of $50 \mathrm{mV} \mathrm{s}^{-1}$.

\section{Electrochemical sensor}

Square wave voltammetry measurements were made over a potential range of -0.5 to $1.0 \mathrm{~V}$ (SCE), with a $5 \mathrm{mV}$ potential step, a $0.1 \mathrm{~V}$ square wave pulse height, a $50 \mathrm{mV} \mathrm{s}^{-1}$ potential sweep speed, frequency of $10 \mathrm{~Hz}$ and a $60 \mathrm{~s}$ pre-conditioning time operated in open-circuit. Aliquots of copper(II) chloride were successively added to the electrolyte solution, producing copper(II) chloride concentrations that varied between 9.9-91 $\mu \mathrm{mol} \mathrm{L}^{-1}$.

\section{Atomic absorption spectroscopy}

Flame atomic absorption spectroscopy (FAAS) experiments were carried out by using a Shimadzu spectrometer, model AA-7000, with an auto-sample model ASC-7000. A copper hollow cathode lamp from Analytik Jena AG was used. The analytical procedure adopted in FAAS analysis was the same described by Soares et al. ${ }^{22}$

\section{Results and Discussion}

Formation of the organic-inorganic hybrid material occurred through hydrolysis of the TEOS and APTMS groups, which produced silanol-type reactive groups. Condensation of the TEOS and APTMS silanol groups with the linear chains of PDMS occurred simultaneously. After the addition of a conductive component a polymeric network with carbon incorporated was obtained as illustrated in an idealized representation at Figure S2 (SI section).

There was no need to use an agglutination agent when preparing the electrode materials containing PDMS (E3, E6, E12 and E18) because the paste was compacted after manually pressing into the disc cavity. Similar situation is observed for carbon ceramic electrodes, which are prepared in a single-step process and put into the electrode without use of agglutinant. ${ }^{23,24}$ Whereas mineral oil was required to fix the paste to the electrode in the E0 electrode, without PDMS, for a direct comparison with proposed materials. In addition, preparation of the material was carried out in a single stage; therefore, there was no need for subsequent mixing during electrode preparation.

\section{Infrared absorption spectroscopy}

Figure 1 shows the infrared region absorption spectra for the synthesized materials. The E0 material, containing no PDMS, displayed absorption bands at 2968 and $2913 \mathrm{~cm}^{-1}$ that correspond to asymmetric stretching $\left(v_{\mathrm{a}}\right)$ and symmetric stretching $\left(v_{\mathrm{s}}\right)$, respectively, of the $\mathrm{C}-\mathrm{H}$ bonds in the $\mathrm{Si}\left(\mathrm{CH}_{2}\right)_{3}$ group. The absorptions at 1474 and $1388 \mathrm{~cm}^{-1}$ correspond to symmetrical angular deformations $\left(\delta_{\mathrm{s}}\right)$ and asymmetrical angular deformation $\left(\delta_{\mathrm{a}}\right)$, respectively, of the same $\mathrm{C}-\mathrm{H}$ bonds. Furthermore, the spectrum shows 
absorption bands at 1649 and $1564 \mathrm{~cm}^{-1}$ corresponding to symmetrical and asymmetrical angular deformation, respectively, of the $\mathrm{N}-\mathrm{H}$ bond in $\mathrm{NH}_{2}$. The bands at 1600 and $3400 \mathrm{~cm}^{-1}$ correspond to $\mathrm{C}-\mathrm{N}$ and $\mathrm{N}-\mathrm{H}$ stretching, respectively, in $\mathrm{NH}_{2}$. The infrared absorption spectra of the E3, E6, E12 and E18 materials, display two bands in the region between 1100 and $1020 \mathrm{~cm}^{-1}$; these bands are due to asymmetric stretching of the $\mathrm{Si}-\mathrm{O}-\mathrm{Si}$ bond and are characteristic of linear polysiloxane chains. This highlights the condensation of TEOS and APTMS during formation of the materials. The band at $768 \mathrm{~cm}^{-1}$ is associated with the $\mathrm{D} 1$ vibration mode (symmetrical stretching of the $\mathrm{Si}-\mathrm{O}-\mathrm{Si}$ bonds) of cyclic siloxane $(\mathrm{Si}-\mathrm{O})_{\mathrm{n}}$ in the silsesquioxane units that exist in the network nodes. ${ }^{6,9}$

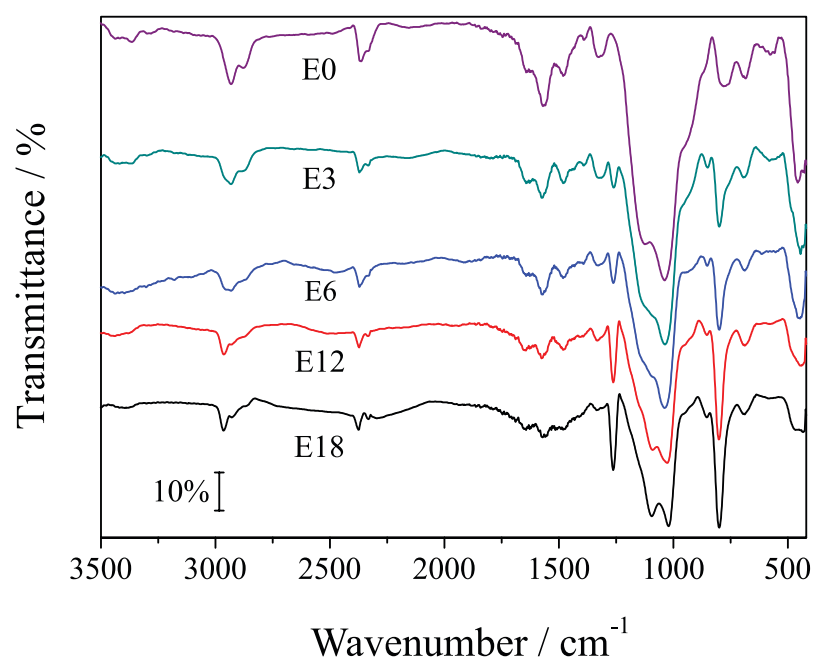

Figure 1. Infrared absorption spectra for the synthesized materials.

\section{Thermogravimetric analysis}

The first mass loss of approximately 3 to $4 \%$ observed in all materials and occurring between $27-180^{\circ} \mathrm{C}$ may be linked to the physical absorption of water and/or residual solvent. The synthesized materials display thermal stability between 350 and $380^{\circ} \mathrm{C}$. In the E0 material, the $396-698^{\circ} \mathrm{C}$ mass loss may be related to degradation of the APTMS propyl groups. ${ }^{9}$ In the thermogravimetric curves of the other materials (E3, E6, E12 and E18), the mass loss between $350-800{ }^{\circ} \mathrm{C}$ may be attributed to decomposition of APTMS with simultaneous polymeric network degradation processes. In the E12 and E18 composites, the PDMS degradation process took place at a slower pace presenting a second peak in the DTG (derivative thermogravimetric analysis) curve (Figure 2). ${ }^{9-11}$

The materials showed high residual mass levels, varying from $77-85 \%$. Since $50 \%$ of the material mass comes from the added graphite, it may be inferred that the polymeric network generated a residual mass between $27-35 \%$, indicating a high degree of PDMS and APTMS reticulation when compared to previous materials, suggesting the formation of a polymeric network of a high degree of silsesquioxane clusters, containing graphite grains into the polymeric network..$^{10,11}$
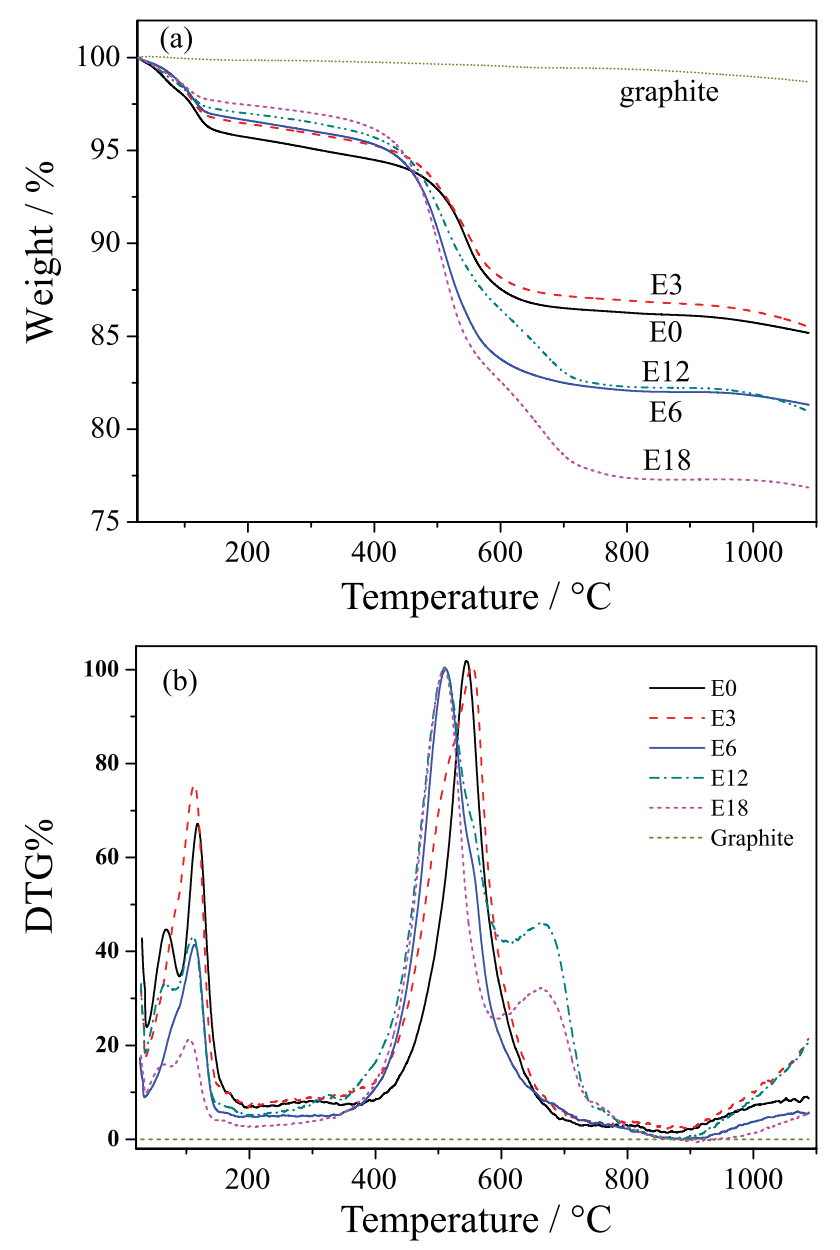

Figure 2. Thermogravimetric analysis of the prepared composites (Table 1): (a) TGA and (b) DTG.

\section{Scanning electron microscopy (SEM)}

Figure 3 shows the SEM micrographs for graphite, E0 and E6. The micrographs show that the graphite and the electrode E0 have a less uniform surface than the E6 electrode. This occurs probably because the PDMS has a smooth flat surface that slightly decreases the roughness of the final material, even in a small amount, as in the electrode E6. ${ }^{25}$ There is no evidence, for magnification used, that there has been phase segregation at the E6 electrode.

\section{Electrochemical studies of the synthesized materials}

Figure 4 shows cyclic voltammograms obtained for the five compositions in the presence of supporting 

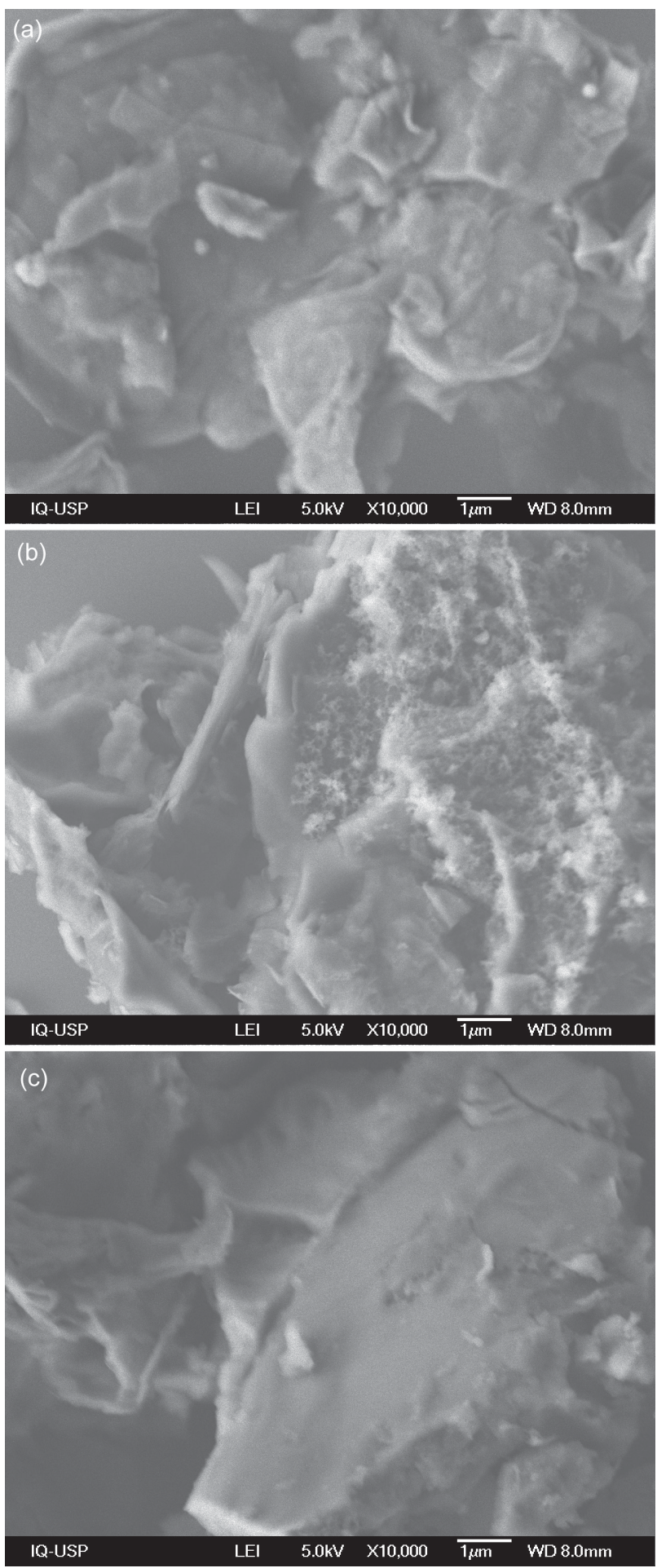

Figure 3. Scanning electronic microscopy images of (a) graphite; (b) E0 and (c) E6.

electrolyte. No faradaic current was observed. The capacitive current decreased as the level of PDMS in the composition of the materials increased. The charged functional group should arrange the charges at the surface, since there are a minor amount of amino in the region of the double layer, this generates a more efficient

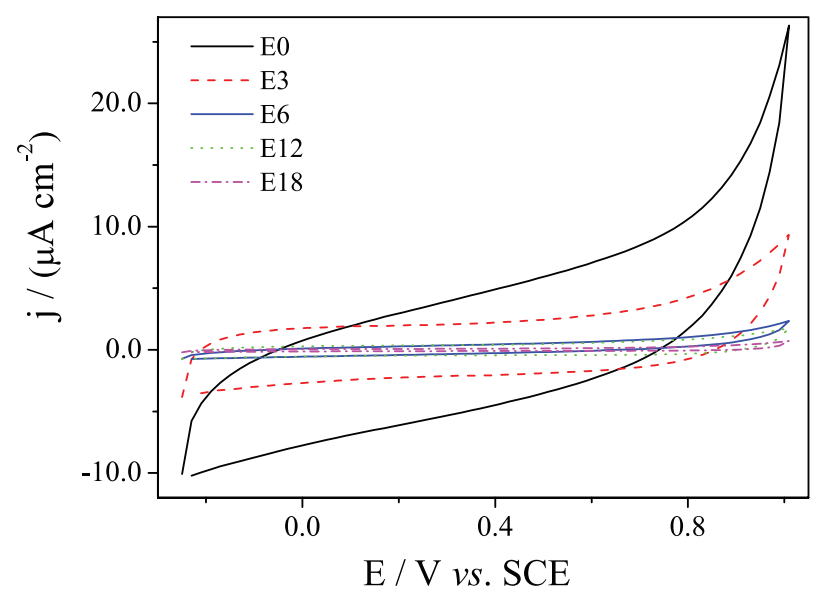

Figure 4. Cyclic voltammograms of the E0, E3, E6, E12 and E18 electrodes with only supporting electrolyte $\left(0.1 \mathrm{~mol} \mathrm{~L}^{-1} \mathrm{KCl}\right)$ and a sweeping speed of $50 \mathrm{mV} \mathrm{s}^{-1}$.

arrangement of electrical charges, therefore reducing the size of the electric double layer. ${ }^{26}$

Studying the redox process of an electroactive species, such as the $\left[\mathrm{Fe}(\mathrm{CN})_{6}\right]^{3-1 / 4}$ redox couple, is a convenient tool to monitor the kinetic barrier to electron transfer at the electrode/solution interface. ${ }^{27}$ Figure S3 (SI section) shows cyclic voltammograms for the five compositions in presence of the $\left[\mathrm{Fe}(\mathrm{CN})_{6}\right]^{3-4-4}$ redox couple at different sweeping speeds.

The anodic and cathodic peak currents were linearly proportional to the square root of the potential sweeping speed highlighting a process controlled by diffusion of the $\left[\mathrm{Fe}(\mathrm{CN})_{6}\right]^{3-14}$ ions to the electrode/solution interface..$^{28}$ Moreover, increasing the potential sweeping speed causes a growing separation between the anodic and cathodic peaks for all electrodes. This indicates a quasi-reversible process. The peak separation and current ratio $\left(\mathrm{I}_{\mathrm{pa}} / \mathrm{I}_{\mathrm{pc}}\right)$ were $0.161 \mathrm{~V}$ (SCE) and 1.074, respectively, for the E6 electrode; and $0.383 \mathrm{~V}$ (SCE) and 1.032, respectively, for the E0 electrode.

The E6 electrode presented a lower capacitive current, peak separation and redox peaks better defined. Among the prepared electrodes the E6 presented a voltammetric response which indicates a surface more organized and, consequently, more adequate to apply for copper detection. Therefore, the E6 electrode was chosen for further investigation, along with the electrode without PDMS for comparison.

The electrochemical stability of the E0 and E6 electrodes was investigated over 100 consecutive cycles. Figure S4 (SI section) shows the cyclic voltammograms obtained from these electrodes: E0 and E6. The results suggest that both electrodes are stable up to the studied interval of 100 cycles. The variation in capacitive current is smaller for the E6 electrode when compared to the E0 electrode. This is probably due to the hydrophobic characteristic of the 
PDMS. At the electrode surface water/potassium chloride should have an inefficient interaction with PDMS chain, reducing the amount of charges, in consequence the double layer. Simultaneously, there are amino groups at the surface, what in opposite, strongly interact with water/potassium chloride and drives to a higher arrangement of the charges. Since the number of amino groups at E6 electrode is lower than in E0, a small double layer was observed. ${ }^{29,30}$

With the aim of providing more electrochemical information about these electrodes, in Figure 5, Bode diagrams for the E0 and E6 electrodes at open circuit potential in $0.1 \mathrm{~mol} \mathrm{~L}^{-1} \mathrm{KCl}$ are shown. The impedance results suggest that the presence of PDMS in the electrode bulk alters the electrochemical behavior of the prepared electrodes. Bode diagram shows that both display a time constant at lower frequencies with the dominant characteristics of a capacitor. Differences in behavior are evident in the presence of PDMS, with a second time constant present at high frequencies in the E6 electrode that indicates a second stage due to a higher electrode resistance caused by the PDMS polymeric network. Furthermore, there is an increase in the polarization resistance of the material. ${ }^{31}$ This is probably due to the insulating characteristics of PDMS.
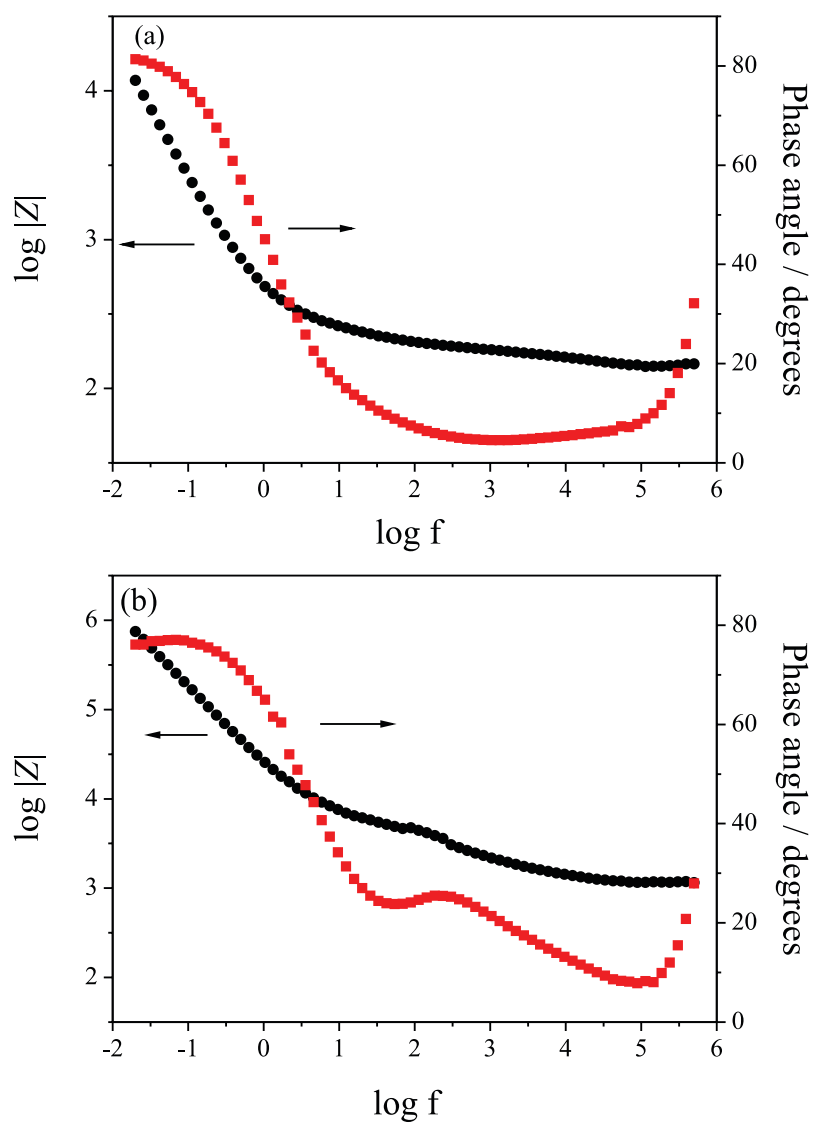

Figure 5. Bode spectra for the (a) E0; (b) E6 electrodes in a $0.1 \mathrm{~mol} \mathrm{~L}^{-1}$ $\mathrm{KCl}$ medium at open circuit potential, with a frequency range of $500 \mathrm{kHz}$ to $20 \mathrm{MHz}$ and a $5 \mathrm{mV}$ sine voltage width.

\section{Detection of copper ion}

A comparison between a graphite electrode and the E6 electrode is presented at Supplementary Information (Figure S5), showing that on carbon there is a superposition of peaks when compared with E6 electrode, which presents better resolved peaks for copper ion. Figure 6 shows square wave voltammograms obtained for the E0 and E6 electrodes with aliquots of a copper(II) ion solution added to the supporting electrolyte.
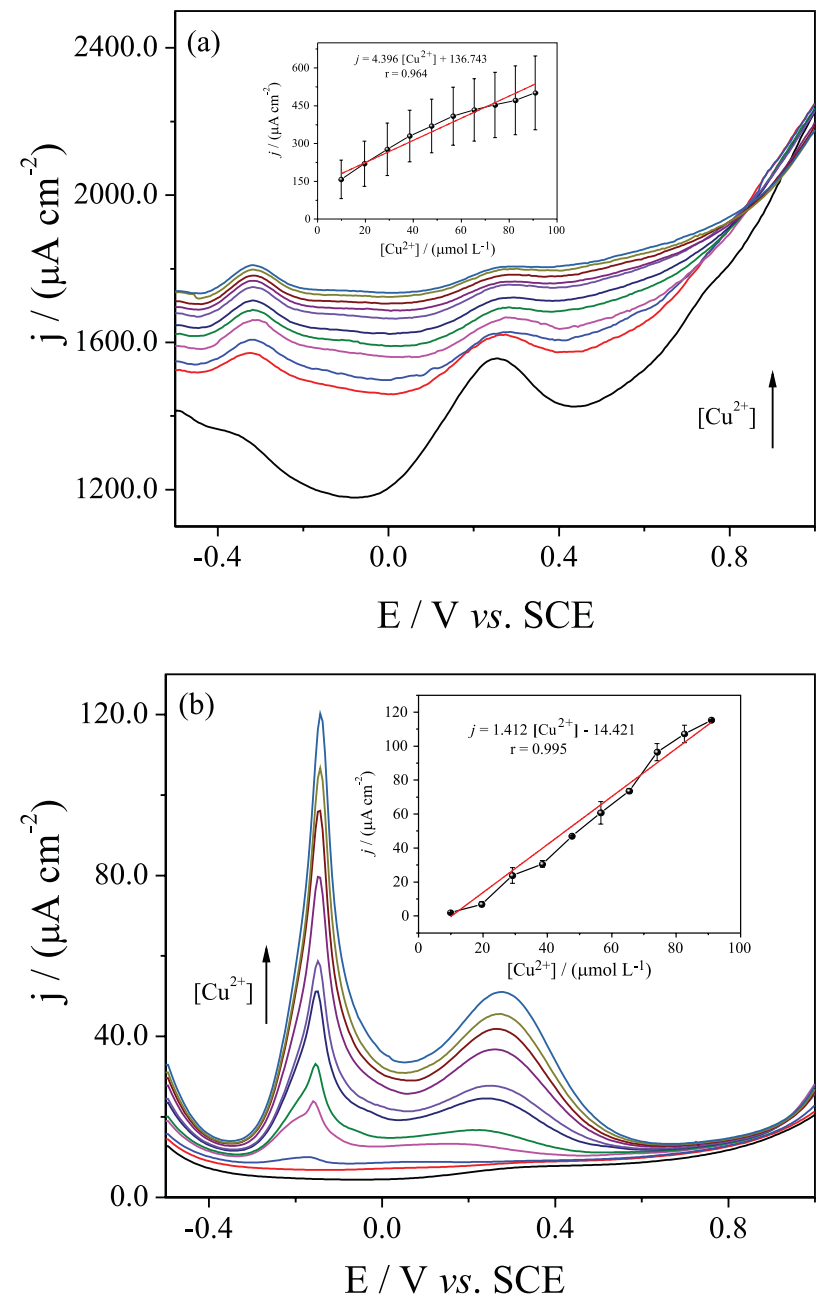

Figure 6. Square wave voltammograms of the (a) E0 and (b) E6 electrodes in a $0.1 \mathrm{~mol} \mathrm{~L}^{-1} \mathrm{KCl}$ medium, containing copper(II) chloride $\left(\mathrm{CuCl}_{2}\right)$ varying in concentration between 9.9-91 $\mu \mathrm{mol} \mathrm{L}^{-1}$, at a potential sweep speed of $50 \mathrm{mV} \mathrm{s}^{-1} ; 5 \mathrm{mV}$ potential step; $0.1 \mathrm{~V}$ square wave pulse height; frequency $10 \mathrm{~Hz}$.

During anodic sweeping, two peaks are observed in the cyclic voltammograms of both electrodes: a peak at approximately $-0.15 \mathrm{~V}$ and another at approximately $0.40 \mathrm{~V}$. The first process may be attributed to the oxidation of copper ions interacting with amino groups in the electrode material. This affirmation is based on 
comparison of the current densities, with the current density in the E0 electrode system greater than that in the E6 electrode system. It is expected that there will be fewer amino groups on the E6 electrode surface due to the condensation of APTMS with PDMS during preparation of the E6 electrode. The second oxidation process, which occurs at a greater potential, is related to the oxidation of copper ions adsorbed in a disordered manner on the electrode surface. ${ }^{15,16,32,33}$

Limit of detection (LOD) and limit of quantification (LOQ) analytical parameters were determined for the quantification of copper ions by both the E0 and E6 electrodes. The data are shown in Table 2.

Table 2. Linear range, limit of detection (LOD) and limit of quantification (LOQ) for the quantification of copper(II) ions by the E0 and E6 electrodes

\begin{tabular}{lccc}
\hline Electrode & $\begin{array}{c}\text { Linear range / } \\
\left(\mathrm{mol} \mathrm{L}^{-1}\right)\end{array}$ & $\begin{array}{c}\text { LOD / } \\
\left(\mathrm{mol} \mathrm{L}^{-1}\right)\end{array}$ & $\begin{array}{c}\text { LOQ / } \\
\left(\mathrm{mol} \mathrm{L}^{-1}\right)\end{array}$ \\
\hline E0 & $9.9 \times 10^{-6}-9.1 \times 10^{-5}$ & $4.6 \times 10^{-5}$ & $1.6 \times 10^{-4}$ \\
E6 & $9.9 \times 10^{-6}-9.1 \times 10^{-5}$ & $1.9 \times 10^{-7}$ & $6.2 \times 10^{-7}$ \\
\hline
\end{tabular}

A comparison of the prepared electrodes with some of those reported in the literature is shown in Table 3.

Square wave voltammetry measurements were obtained in presence of different metals to evaluate the E6 selectivity for the ion determination $\mathrm{Cu}^{2+}$. As can be observed at Figure S6 (SI section), in the presence of $\mathrm{Zn}^{2+}, \mathrm{Co}^{2+}$ and $\mathrm{Ni}^{2+}$ ions with successive increase of concentration, no peak for the interferents studied in the potential range was observed, only the peaks referring to the copper ions. This result indicates a high selectivity of the electrode proposed for the determination of copper.

The voltammetry technique and pre-concentration time will change the sensitivity of the copper ion detection, in the
Table 3 it was presented a similar CMEs with significantly higher signals than those recorded by the E6 electrode. But as the main purpose of this paper was the insertion of an insulate polymer and only investigate the uptake properties of the proposed material, the concentration range obtained with linear sweep stripping voltammetry was fully satisfactory. In addition, the results were obtained with a lower time of pre-concentration when compared with other electrodes in Table 3, with the advantage of not using the binder.

\section{Determination of copper in sugarcane spirit}

Electrode E6 was applied in analytical determination of real sample, sugarcane spirit. Figure 7 shows the voltammograms obtained for the E6 electrode through the square wave voltammetry technique. The measurements

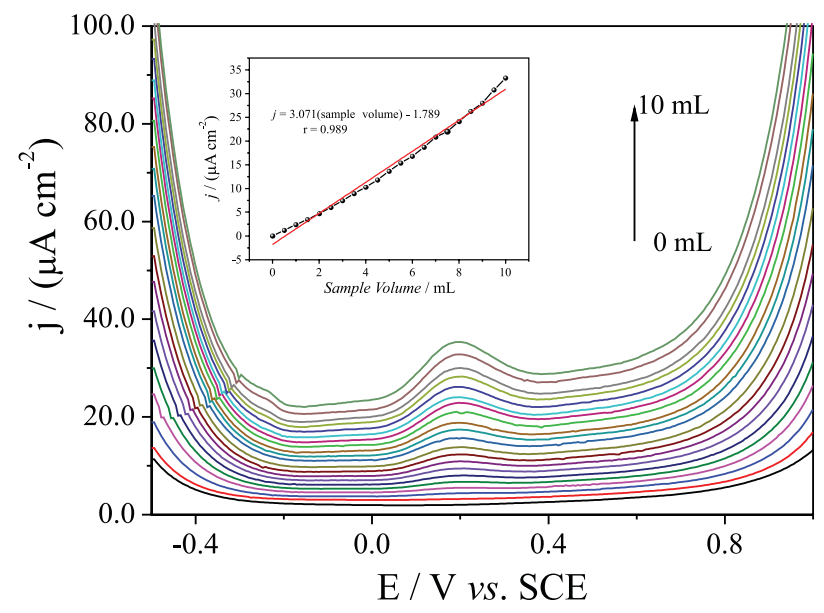

Figure 7. Square wave voltammograms of the E6 electrodes in a $0.1 \mathrm{~mol} \mathrm{~L}^{-1} \mathrm{KCl}$ medium, containing sugarcane spirit, varying in sample volume from 0 to $10 \mathrm{~mL}$ at a potential sweep speed of $50 \mathrm{mV} \mathrm{s}^{-1}$, with a $5 \mathrm{mV}$ potential step, a $0.1 \mathrm{~V}$ square wave pulse height, frequency $10 \mathrm{~Hz}$.

Table 3. Detection on $\mathrm{Cu}^{2+}$ ions with $\mathrm{CMEs}$ reported in the literature

\begin{tabular}{|c|c|c|c|c|c|}
\hline CME & Electro-chemical technique & $\begin{array}{c}\text { Pre-concentration } \\
\text { time / s }\end{array}$ & Binder & Linear range / $\left(\mathrm{mol} \mathrm{L}^{-1}\right)$ & $\begin{array}{c}\mathrm{LOD} / \\
\left(\mathrm{mol} \mathrm{L}^{-1}\right) \\
\end{array}$ \\
\hline $\begin{array}{l}\text { PDMS polymeric network } \\
\text { functionalized with APTMS (E6) }\end{array}$ & $\begin{array}{l}\text { carbon paste } \\
\text { (square wave voltammetry) }\end{array}$ & 60 & no & $\begin{array}{l}9.9 \times 10^{-6}-3.8 \times 10^{-5} \\
3.8 \times 10^{-5}-9.1 \times 10^{-5}\end{array}$ & $1.2 \times 10^{-6}$ \\
\hline $\begin{array}{l}\text { Amino-functionalized mesoporous } \\
\text { silica }^{34}\end{array}$ & $\begin{array}{l}\text { glassy carbon } \\
\text { (linear sweep voltammetry) }\end{array}$ & 240 & yes & $7.8 \times 10^{-11}-1.6 \times 10^{-8}$ & $1.4 \times 10^{-11}$ \\
\hline Printed polymer (4-vinylpyridine) ${ }^{32}$ & $\begin{array}{c}\text { carbon paste } \\
\text { (differential pulse voltammetry) }\end{array}$ & 4800 & yes & $\begin{array}{l}7.0 \times 10^{-8}-1.0 \times 10^{-6} \\
1.0 \times 10^{-6}-1.0 \times 10^{-4}\end{array}$ & $2.3 \times 10^{-8}$ \\
\hline Chitosan $^{3}$ & $\begin{array}{c}\text { carbon paste } \\
\text { (anodic dissolution voltammetry) }\end{array}$ & 270 & yes & $2.0 \times 10^{-7}-7.4 \times 10^{-6}$ & $8.3 \times 10^{-8}$ \\
\hline $\begin{array}{l}\text { Silica gel organofunctionalized } \\
\text { with } 2 \text {-aminothiazole } 35\end{array}$ & $\begin{array}{c}\text { carbon paste } \\
\text { (differential pulse anodic stripping } \\
\text { voltammetry) }\end{array}$ & 1200 & yes & $7.5 \times 10^{-8}-2.5 \times 10^{-6}$ & $3.1 \times 10^{-8}$ \\
\hline $\begin{array}{l}\text { Silica gel organofunctionalized } \\
\text { with 2-benzothiazolethiol }\end{array}$ & $\begin{array}{c}\text { carbon paste } \\
\text { (differential pulse anodic stripping) }\end{array}$ & 180 & yes & $8.0 \times 10^{-7}-1.0 \times 10^{-5}$ & $2.0 \times 10^{-7}$ \\
\hline
\end{tabular}

CME: chemically modified electrodes; PDMS: polydimethylsiloxane; APTMS: 3-aminopropyl-trimethoxysilane; LOD: limit of detection. 
were performed with the same parameters described earlier. Aliquots of the sample were successively added to the $\mathrm{KCl}$ solution, ranging from 0.5 to $10 \mathrm{~mL}$.

The peak related to the presence of the $\mathrm{Cu}^{\mathrm{II}}$ ions in the sugarcane spirit appears at $0.2 \mathrm{~V}$ and it can be observed that increases linearly with higher amount of the aliquot. The concentration of copper in the sample was determined by the current density for a $10 \mathrm{~mL}$ aliquot, which was interpolated on the analytical curve obtained with the standard solution. The value found was $33 \mu \mathrm{mol} \mathrm{L}{ }^{-1}\left(2.1 \mathrm{mg} \mathrm{L}^{-1}\right)$, which is below the maximum limit established (5.0 $\mathrm{mg} \mathrm{L}^{-1}$ of copper) in sugarcane spirit, according to the Brazilian legislation (MAPA)..$^{37,38}$ The value obtained for E6 was compared with the concentration determined by the reference technique, flame atomic absorption, which was $2.48 \mathrm{mg} \mathrm{L}^{-1}$ of copper, obtaining a relative error of 0.15 .

\section{Conclusions}

The percentage of PDMS in the polymeric network of the electrode materials altered their electrochemical behavior. The results suggest that electron transfer at the electrode/solution interface with the redox probe $\mathrm{K}_{3} \mathrm{Fe}(\mathrm{CN})_{6}$ is controlled by diffusion of the probe ions in solution. The electrodes displayed electrochemical stability and a voltammetric profile with a broad potential window. Electrochemical impedance measurements showed that insertion of PDMS into the polymeric network of the electrodes provokes an increase in their polarization resistance that does not harm electron transfer processes. A PDMS-induced decrease in current densities during copper(II) ion oxidation was mostly attributed to a reduction in the number of amino groups on the electrode surface. However, the concentration of copper in a real sample was adequately obtained on the electrode E6.

In this work, it was demonstrated that a material based on PDMS polymeric network can be used as a chemical modifier to obtain an electrode with a reproducible electrochemical response and potential to be applied as electrochemical sensors for detection of copper ions in aqueous solution. In addition, due to the PDMS characteristics, to prepare these electrodes there was no necessity to add an agglutinating agent, as usually is necessary in carbon modified electrode to achieve a mechanical stable paste.

\section{Supplementary Information}

Supplementary information is available free of charge at http://jbcs.org.br as PDF file.

\section{Acknowledgments}

The authors are indebted to CAPES and FAPEMIG for the R. P. D. S. master's fellowship, A. M. S. L. to CAPES for postdoctoral fellow funding and F. L. P. to CNPq for financial support.

\section{References}

1. Souza, M. F. B.; Quim. Nova 1997, 20, 191.

2. Bellido-Milla, D.; Cubillana-Aguilera, L. M.; El Kaoutit, M.; Hernández-Artiga, M. P.; de Cisneros, J. L. H.-H.; NaranjoRodríguez, I.; Palacios-Santander, J. M.; Anal. Bioanal. Chem. 2013, 405, 3525.

3. Janegitz, B. C.; Marcolino Junior, L. H.; Fatibello-Filho, O.; Quim. Nova 2007, 30, 1673.

4. Lowinsohn, D.; Bertotti, M.; Quim. Nova 2006, 29, 1318.

5. Crespilho, F. N.; Rezende, M. O. O.; Quim. Nova 2004, 27, 964.

6. Oktay, B.; Kayaman-Apohan, N.; J. Coat. Technol. Res. 2013, 10,785 .

7. Lei, K. F.; Lee, K. F.; Lee, M.-Y.; Microsyst. Technol. 2013, 20, 1351.

8. Soulas, D. N.; Sanopoulou, M.; Papadokostaki, K. G.; Mater. Sci. Eng, C 2013, 33, 2122.

9. Silva, F. A. B.; Pissetti, F. L.; J. Colloid Interface Sci. 2014, $416,95$.

10. Pissetti, F. L.; Magosso, H. A.; Yoshida, I. V.; Gushikem, Y.; Myernyi, S. O.; Kholin, Y. V.; J. Colloid Interface Sci. 2007, 314, 38.

11. Pissetti, F. L.; Yoshida, I. V. P.; Gushikem, Y.; Kholin, Y. V.; Colloids Surf., A 2008, 328, 21.

12. Pissetti, F. L.; de Araújo, P. L.; Silva, F. A. B.; Poirier, G. Y.; J. Braz. Chem. Soc. 2015, 26, 266.

13. Saensak, R.; Faibut, N.; Porntheeraphat, S.; Paosawatyanyong, B.; Amornkitbamrung, V.; Triroj, N.; Procedia Eng. 2012, 47, 374.

14. Gökçeören, A. T.; Erbil, C.; Surf. Interface Anal. 2011, 43, 1403.

15. Ko, Y. G.; Lee, H. J.; Oh, H. C.; Choi, U. S.; J. Hazard. Mater. 2013, 260, 489.

16. Yang, G.-X.; Jiang, H.; Water Res. 2014, 48, 396.

17. Nagarale, R. K.; Lee, J. M.; Shin, W.; Electrochim. Acta 2009 , $54,6508$.

18. Saadeh, S. M.; Abu-Shawish, H. M.; Abed-Almonem, K. I.; Baraka, A.; abu Mkhada, A.; Safi, W.; J. Electroanal. Chem. 2012, 687, 11.

19. Peng, C.; Jin, R.; Li, G.; Li, F.; Gu, Q.; Sep. Purif. Technol. 2014, 136, 42.

20. Uriu-Adams, J. Y.; Keen, C. L.; Mol. Aspects Med. 2005, 26, 268.

21. Demirel, G.; Birlik, G.; Çakmak, M.; Çaykara, T.; Ellialtığlu, Ş.; Surf. Sci. 2007, 601, 3740. 
22. Soares, I. V.; Vieira, E. G.; do Carmo, D. R.; Dias Filho, N. L.; Microchem. J. 2013, 110, 120.

23. Módolo, M. L.; Valandro, S. R.; Pessoa, C. A.; Fujiwara, S. T.; Electrochim. Acta 2013, 112, 783.

24. Razmi, H.; Habibi, E.; Heidari, H.; Electrochim. Acta 2008, 53, 8178.

25. Yazdanbakhsh, F.; Alizadehgiashi, M.; Sawada, J. A.; Kuznicki, S. M.; Water Sci. Technol. 2016, 73, 1409.

26. Luechinger, M.; Prins, R.; Pirngruber, G. D.; Microporous Mesoporous Mater. 2005, 85, 111.

27. de Morais, A.; Silveira, G.; Villis, P. C. M.; Maroneze, C. M.; Gushikem, Y.; Pissetti, F. L.; Lucho, A. M. S.; J. Solid State Electrochem. 2012, 16, 2957.

28. Bard, A.; Faulkner, L.; Russ. J. Electrochem. 2002, 38, 1505.

29. Panou, A. I.; Papadokostaki, K. G.; Tarantili, P. A.; Sanopoulou, M.; Eur. Polym. J. 2013, 49, 1803.

30. Stanton, M. M.; Ducker, R. E.; MacDonald, J. C.; Lambert, C. R.; Grant McGimpsey, W.; J. Colloid Interface Sci. 2012, 367, 502.
31. Huang, J.; Li, Z.; Liaw, B. Y.; Zhang, J.; J. Power Sources 2016, 309,82 .

32. Zhihua, W.; Xiaole, L.; Jianming, Y.; Yaxin, Q.; Xiaoquan, L.; Electrochim. Acta 2011, 58, 750.

33. Takeuchi, R. M.; Santos, A. L.; Medeiros, M. J.; Stradiotto, N. R.; Microchim. Acta 2009, 164, 101.

34. Dai, X.; Qiu, F.; Zhou, X.; Long, Y.; Li, W.; Tu, Y.; Anal. Chim. Acta 2014, 848, 25.

35. Takeuchi, R.; Santos, A.; Padilha, P.; Stradiotto, N.; Talanta 2007, 71, 771.

36. Marino, G.; Matos, J. R.; Cavalheiro, E. T. G.; Ecletica Quim. 2007, 32, 29.

37. Lima, A. J. B.; Cardoso, M. G.; Pimentel, M. C. G. F. A.; Quim. Nova 2006, 29, 247.

38. Costa, D. A.; Takeuchi, R. M.; Santos, A. L.; Int. J. Electrochem. Sci. 2011, 6, 6410.

Submitted: August 3, 2017

Published online: March 16, 2018 